\title{
Sox4 drives Atoh1-independent intestinal secretory differentiation toward tuft and enteroendocrine fates
}

Adam D Gracz ${ }^{1 *}$, Leigh Ann Samsa ${ }^{2}$, Matthew J Fordham², Danny C Trotier ${ }^{2}$, Bailey Zwarycz ${ }^{3}$, Yuan-Hung Lo ${ }^{4}$, Katherine Bao ${ }^{5}$, Joshua Starmer ${ }^{1}$, Noah F Shroyer ${ }^{4}$, R. Lee Reinhardt ${ }^{5} \dagger$, Scott T Magness $s^{2,3,6^{*}}$

\author{
Affiliations: \\ ${ }^{1}$ Department of Genetics, University of North Carolina at Chapel Hill, Chapel Hill, NC \\ ${ }^{2}$ Department of Medicine, University of North Carolina at Chapel Hill, Chapel Hill, NC \\ ${ }^{3}$ Department of Cell Biology \& Physiology, University of North Carolina at Chapel Hill, \\ Chapel Hill, NC \\ ${ }^{4}$ Department of Medicine, Section of Gastroenterology and Hepatology, Baylor College \\ of Medicine, Houston, TX \\ ${ }^{5}$ Department of Immunology, Duke University, Durham, NC \\ ${ }^{6}$ Joint Department of Biomedical Engineering, University of North Carolina at Chapel \\ Hill/North Carolina State University, Chapel Hill, NC
}

*Co-correspondending authors:

Adam D. Gracz: agracz@med.unc.edu

Scott T. Magness: magness@med.unc.edu

$\dagger$ Current address: Department of Biomedical Research National Jewish Health and Department of Immunology and Microbiology, University of Colorado School of Medicine, Denver CO.

The authors declare no conflicts of interest

Author contributions:

A.D.G. and S.T.M. designed the project and wrote the manuscript. A.D.G., L.A.S., M.J.F., D.C.T., and B.Z. carried out experiments. A.D.G. and J.S. carried out RNA-seq analysis and J.S. and S.T.M. generated t-SNE plots from scRNA-seq data. K.B. and R.L.R. consulted on and carried out helminth experiments. Y.L. and N.F.S. provided Atoh1 ${ }^{\text {GFP }}$ tissues. S.T.M. supervised research. 


\section{Background \& Aims}

The intestinal epithelium is maintained by intestinal stem cells (ISCs), which produce post-mitotic absorptive and secretory epithelial cells. Initial fate specification toward enteroendocrine, goblet, and Paneth cell lineages is dependent on Atoh1, a master regulator of secretory differentiation. However, the origin of tuft cells, which participate in Type II immune responses to parasitic infection, is less clear and appears to occur in an Atohl-independent manner. Here we examine the role of Sox4 in ISC proliferation and differentiation.

\section{Methods}

We used mice with intestinal epithelial-specific conditional knockout of Sox4 $\left(\mathrm{Sox} 4^{\mathrm{fl} / \mathrm{fl}}\right.$ :vilCre; Sox $\left.4 \mathrm{cKO}\right)$ to study the role of Sox4 in the small intestine. Crypt- and single cell-derived organoids were used to assay proliferation and ISC potency between control and Sox4cKO mice. Lineage allocation and genetic consequences of Sox 4 ablation were studied by immunofluorescence, RT-qPCR, and RNA-seq. In vivo infection with helminths and in vitro cytokine treatment in primary intestinal organoids were used to assess tuft cell hyperplasia in control and Sox $4 \mathrm{cKO}$ samples. Atoh $1^{\text {GFP }}$ reporter mice and single cell RNA-seq (scRNA-seq) were used to determine co-localization of SOX4 and Atoh1. Wild-type and inducible Atohl knockout (Atoh $1{ }^{\mathrm{fl} / \mathrm{fl}}$ :vilCreER; Atoh1iKO) organoids carrying an inducible Sox4 overexpression vector (Sox4OE) were used to determine the role of Atohl in Sox 4 driven secretory differentiation.

\section{Results}

Loss of Sox4 impairs ISC function and secretory differentiation, resulting in decreased numbers of enteroendocrine and tuft cells. In wild-type mice, SOX4+ cells are significantly upregulated following helminth infection coincident with tuft cell hyperplasia. Sox4 is activated by IL13 in vitro and Sox4cKO knockout mice demonstrate impaired tuft cell hyperplasia and parasite clearance following infection with helminths. A subset of Sox4-expressing cells colocalize with Atoh1 and enteroendocrine markers by scRNA-seq, while Sox4+/Atoh1- cells correlate strongly with tuft cell populations. Gainof-function studies in primary organoids demonstrate that Sox4 is sufficient to drive both enteroendocrine and tuft cell differentiation, and can do so in the absence of Atohl.

\section{Conclusion}

Our data demonstrate that Sox 4 promotes enteroendocrine and tuft cell lineage allocation independently of Atoh1. These results challenge long-standing views of Atohl as the sole regulator of secretory differentiation in the intestine and are relevant for understanding host epithelial responses to parasitic infection.

Keywords: intestinal stem cells; differentiation; tuft cells 


\section{Introduction}

The intestinal epithelium is essential for both digestive and barrier function and contains a diverse complement of post-mitotic cells which carry out complex physiological functions critical for homeostasis. Functional cell types in the intestine can be broadly subdivided into: (a) absorptive enterocytes and (b) secretory lineages, which include antimicrobial-producing Paneth cells, mucus-producing goblet cells, and hormone-secreting enteroendocrine cells (EECs) ${ }^{1}$. Tuft cells, which represent a less wellcharacterized lineage, initiate immune responses to parasitic infections in the intestine ${ }^{2-}$ ${ }^{5}$.With the exception of long-lived Paneth cells, these post-mitotic lineages are subject to the rapid (5-7 day) and continuous turnover of the intestinal epithelium. Therefore, their numbers must be maintained through constant proliferation and differentiation of the intestinal stem cells (ISCs), which reside in a specialized stem cell niche at the base of the intestinal crypts.

Extrinsic signals from the niche induce regulatory programs in ISCs and their immediate progeny, transit-amplifying progenitor cells (TAs), to drive commitment to different cellular lineages. For example, loss of Notch signaling in ISCs and early progenitors is associated with the induction of secretory differentiation through the derepression of Atoh1, which is widely regarded as the master regulator of secretory fate 6, 7. Certain pathological settings can shift lineage allocation in the intestine, as exemplified by goblet and tuft cell hyperplasia in the setting of type II immune responses. While tuft cells are also derived from ISCs, the transcriptional regulatory mechanisms governing their differentiation and hyperplastic response to infection remain less well characterized ${ }^{3}$. This is due in part to evidence that tuft cells differentiate independently of Atoh1, but still appear to require downregulation of Notch ${ }^{6,8,9}$. Recent studies have demonstrated upregulation of tuft cell numbers following Atohl deletion in vivo, and systems biology approaches have supported an Atohl-independent differentiation program for this lineage ${ }^{10}$. Together, these data suggest an apparently paradoxical Notchrepressed, Atohl-independepent mechanism for tuft cell specification.

Sry-box containing (Sox) factors are transcription factors with broad regulatory roles in stem cell maintenance and differentiation in many adult tissues, including the intestinal epithelium ${ }^{11,12}$. Sox 9 is known to contribute to Paneth cell differentiation, and previous work from our group has demonstrated that distinct expression levels of Sox 9 mark stem and progenitor cell populations from intestinal crypts, including labelretaining cells (LRCs) ${ }^{13-16}$. The role of other Sox factors in ISC function and differentiation remain uncharacterized.

Sox 4 has been associated with the ISC genomic signature and is correlated with increased invasion/metastasis and decreased survival in colon cancer, but its role in intestinal epithelial homeostasis is unknown ${ }^{17,18}$. In the hematopoietic system, as well as the developing nervous system, pancreas, kidney, and heart, Sox4 is required for both proper cell lineage allocation and maintenance of progenitor pools ${ }^{19-21}$. The diverse regulatory potential of Sox4, coupled with its known role in a broad range of tissuespecific progenitor populations, led us to hypothesize that it may function as a regulator of ISC maintenance and differentiation. In this study we establish a role for Sox4 in ISC homeostasis and regulation of tuft cell hyperplasia during parasitic helminth infection, which represents a novel pathway for tuft cell specification with important implications for human responses to parasitic disease. 


\section{Results}

\section{SOX4 is expressed at distinct levels in the ISC zone}

To characterize Sox 4 expression, we first used in situ hybridization to detect Sox 4 mRNA. Consistent with previous reports, Sox 4 is restricted to the base of the intestinal crypts in the stem cell zone (Figure 1A) ${ }^{18}$. Since the cellular resolution of in situ hybridization is low, we leveraged a previously characterized $\operatorname{Sox} 9^{E G F P}$ reporter mouse to examine the expression of Sox4 in different crypt cell types. Distinct Sox $9^{E G F P}$ levels differentially mark active ISCs (Sox $9^{\text {low }}$ ), secretory progenitors/reserve ISCs $\left(\right.$ Sox $\left.9^{\text {high }}\right)$, TA progenitors $\left(\right.$ Sox $\left.9^{\text {sublow }}\right)$, Paneth cells $\left(\right.$ Sox $9^{\text {EGFP-neg. }}$ CD $\left.24^{+}\right)$, and non-Paneth postmitotic cells $\left(\right.$ Sox $\left.9^{E G F P-n e g}: \mathrm{CD} 24^{\text {neg }}\right){ }^{13,14,22,23}$. Sox $9^{E G F P}$ populations were isolated by fluorescence-activated cell sorting (FACS) and analyzed by RT-qPCR. Sox4 was upregulated in two populations: Sox $9^{\text {low }}$, which is consistent with active ISCs, and Sox $9^{\text {high }}$, which is consistent with secretory progenitors (Figure 1B) ${ }^{23,24}$.

Next, we examined SOX4 protein expression by immunofluorescence. SOX4 protein was expressed in a more restricted pattern relative to mRNA. Like Sox9, we observed distinct expression levels of SOX4, which we characterized as "low" and "high" (Figure 1C). SOX 4 low cells were associated with the crypt base columnar (CBC), active ISC population, in cell positions $+1-3$ counting from the base of the crypt (Figure $1 C, \mathrm{D})$. Interestingly, SOX $4^{\text {high }}$ cells were predominantly found in the supra-Paneth cell position, which is commonly associated with early secretory progenitors, at cell positions +4-7 (Figure 1C, D) ${ }^{25}$. Unlike SOX9, which is expressed in villus-based EECs/tuft cells, we did not detect any Sox 4 mRNA or protein outside of the crypts ${ }^{13}$.

Since Sox $9^{\text {high }}$ cells are known to represent EEC-like secretory progenitors, we asked if the SOX $4^{\text {high }}$ population overlapped with Sox $9^{\text {high }}$ cells. However, we found no co-localization between high levels of SOX4 protein and high levels of Sox 9 mRNA, as determined by $\operatorname{Sox} 9^{E G F P}$, which is an accurate surrogate of endogenous $\operatorname{Sox} 9$ mRNA and protein expression ( $n=3$ mice, $100 \mathrm{SOX} 4+$ cells per mouse) (Figure 1E, E') ${ }^{13,26}$. Sox $9^{\text {high }}$-expressing cells are consistent with a subset of label-retaining reserve ISCs (LRCs) located in the supra-Paneth cell position. However, not all LRCs are Sox $9^{\text {high }}{ }^{23}$. We reasoned that the supra-Paneth cell location of SOX $4{ }^{\text {high }}$ cells suggests that they may represent the non-Sox $9^{\text {high }}$ subset of LRCs. To examine this, we counted the number of SOX $4^{\text {high }}$ cells co-localizing with LRCs, as determined by 28 days of continuous labeling with 5-ethynyl-2-deoxyuridine (EdU), followed by 10 days of washout ${ }^{23,25}$. Surprisingly, we found that no SOX $4{ }^{\text {high }}$ cells were LRCs ( $n=3$ mice, 100 SOX4+ cells per mouse) (Figure 1F). We observed rare co-localization between DCKL1, a tuft cell/reserve ISC biomarker also associated with the +4 position, and SOX $4(5.0 \% \pm 3.0 \%$; Figure $1 \mathrm{G})$. Finally, we addressed the possibility that $\mathrm{SOX} 4{ }^{\text {high }}$ cells may represent a quiescent population by co-staining with KI67, which marks all proliferating cells. $90.6 \pm 3.2 \%$ of SOX $4^{\text {high }}$ cells were found to be proliferative (Figure $1 \mathrm{H}$ ). Together, these data suggest that $\mathrm{SOX} 4^{\text {high }}$ cells represent ISCs or progenitors, and are distinct from LRC Sox $9^{\text {high }}$ secretory precursors. Co-localization with DCLK1 suggests that a subset of SOX $4^{\text {high }}$ cells may represent tuft cell or non-LRC secretory precursors.

\section{Loss of Sox4 results in increased proliferation and decreased ISC function}

Complete deletion of Sox4 results in embryonic lethality ${ }^{27}$. To enable 
examination of Sox4 function in adult intestinal epithelium, we crossed previously generated Sox $4^{f l f l}$ mice to mice expressing Cre recombinase driven by the villin promoter (vilCre), which is constitutively expressed in the intestinal epithelium beginning at E13.5 28, 29. To examine Sox4 expression, we isolated CD44- and CD44+ populations from control and Sox4cKO adult intestinal epithelium (CD326+) by FACS and confirmed CD44 enrichment by qPCR (Supplementary Figure 1A-C). CD44 marks crypt cells as well as EECs in the villi (Supplementary Figure 1A). As expected, control animals exhibited significant enrichment of Sox4 in the CD44+ population, consistent with restricted expression of Sox4 in the intestinal crypts (Supplementary Figure 2A). Sox 4 cKO animals did not express detectable levels of Sox4 mRNA or protein (Supplementary Figure 2A, B).

To assay the role of Sox4 in ISC/TA proliferation, we examined KI67 and EdU incorporation, which was administered as a single, $2 \mathrm{hr}$ pulse. Sox $4 \mathrm{cKO}$ crypts exhibited significantly higher percentages of total proliferating cells as well as cells in S-phase (Figure 2A, B). This increase in proliferation was reflected by increased crypt length in Sox 4 cKO intestines (Supplementary Figure 2C). In organoid cultures, Sox4cKO crypts grew at the same rate as control crypts, suggesting that hyperproliferation in the absence of Sox 4 may be masked by exogenous growth factors required for organoid culture (Figure 2C).

Due to its known role in maintenance of stem/progenitor pools in other tissues, we sought to determine if Sox4 regulates the size or function of the ISC pool. OLFM4, which is an established marker of ISCs, was detected by immunofluorescence to assess ISC numbers, which were unchanged between control and Sox $4 \mathrm{cKO}$ intestines (Figure 2D) ${ }^{30}$. Next, we FACS-isolated CD44- and CD44+ cells from control and Sox4cKO mice and conducted single cell organoid forming assays to test ISC function ${ }^{31}$. As expected, CD44- cells failed to form organoids regardless of Sox4 status (Figure 2E). CD44+ cells from Sox $4 \mathrm{cKO}$ mice produced approximately 50\% fewer organoids than controls, demonstrating a significant loss of stemness (Figure 2E).

To determine if this functional deficit was coincident with an increase in apoptosis in Sox4cKO samples, we examined cleaved-caspase-3 expression in the crypts of control and Sox4cKO intestines, as well as the number of apoptotic cells in CD44- and CD44+ populations of cells prepared for FACS. In both cases, we found no significant difference in apoptosis between control and Sox4cKO samples (Supplementary Figure $3 \mathrm{~A}, \mathrm{~B})$. However, CD44+ cells from Sox4cKO mice expressed significantly lower levels of Lgr5, consistent with decreased stem cell function (Figure 2F). Together, these data indicate that Sox 4 does not regulate ISC numbers or apoptosis, but is required for normal ISC function and $\operatorname{Lgr} 5$ expression in the intestinal crypt.

\section{Sox4 regulates secretory lineage allocation}

We next sought to determine if Sox4 regulates differentiation and intestinal lineage allocation. Goblet cells, detected by expression of MUC2, were found at similar numbers between Sox $4 \mathrm{cKO}$ and control intestines (Figure 3A). $\mathrm{CHGA}^{+}$EECs were found in significantly reduced numbers in the villi of Sox $4 \mathrm{cKO}$ mice (Figure 3B). Numbers of $\mathrm{DCLK}^{+}$tuft cells were significantly reduced in crypt and villus compartments of Sox $4 \mathrm{cKO}$ intestines (Figure 3C). Paneth cells were increased in 
Sox4cKO intestines (Figure 3D), reflecting additional Paneth cells at higher positions in Sox $4 \mathrm{cKO}$ crypts (Supplementary Figure 4). Staining patterns for sucrose isomaltase (SIS), a brush-border enzyme associated with absorptive enterocytes, were unchanged between control and Sox4cKO samples (Supplementary Figure 5A). Additionally, villus height was unchanged, and no change was observed for Hes1, which specifies absorptive enterocyte fate (Supplementary Figure 2C and Supplementary Figure 5B).

In order to examine gene regulatory changes in Sox $4 \mathrm{cKO}$ samples in an unbiased manner, we subjected FACS isolated $\mathrm{CD}_{4} 4^{-}$and $\mathrm{CD} 44^{+}$populations to RNA-seq. Consistent with histological observations, decreased expression of genes associated with EEC subpopulations, including pro-EEC transcription factors $I s l 1, N k x 2.2$, and Pax6, was the predominant change observed in Sox $4 \mathrm{cKO} \mathrm{CD}_{4} 4^{+}$populations (Supplementary Figure 1D and Supplementary Table 1) ${ }^{32,33}$. There were no significant gene expression changes in the CD44- populations consistent with the restricted expression pattern of SOX4 in CD44 ${ }^{+}$intestinal crypts and CD44+ status of EECs (Supplementary Figure 1D). Since the EEC lineage is comprised of a diverse complement of subpopulations, we reexamined Sox $4 \mathrm{cKO}$ intestines for the presence of hormones produced by specific EEC subtypes. Compared to the modest reduction in $\mathrm{CHGA}^{+}$cell numbers, we found substantial and significant decreases in EEC subpopulations expressing GIP, CCK, SST, GCG, PYY, and GLP-2 (Figure 3E). Interestingly, numbers of 5-HT and SUBPexpressing populations remained unchanged (Figure 3E).

Since Notch repression is required for EEC specification, we asked if Sox4 is activated following downregulation of Notch. To do so, we treated wild type organoids with the gamma secretase inhibitor DAPT. Since Sox 4 has been reported to be regulated by Wnt in colon cancer cell lines and inhibited by Bmp in hair follicle progenitors, we also treated organoids with WNT3A and Noggin, a Bmp antagonist 34,35. While Sox4 levels and cell numbers did not respond to Wnt activation or Bmp inhibition, we found that Notch inhibition drove a 2 -fold increase in Sox4 expression that coincided with decreased expression of $A s c l 2$, and ISC marker, and increased expression of $\mathrm{Ngn3}$, and early EEC transcription factor (Supplementary Figure 6). Together, these data demonstrate that Sox4 is required for proper secretory lineage allocation. Furthermore, Sox4 is upregulated following Notch inhibition, consistent with early secretory differentiation responses, and required for proper specification of EEC subpopulations.

\section{Sox4 contributes to tuft cell differentiation and hyperplasia}

Since a subset of SOX $4^{\text {high }}$ cells were found to co-localize with DCLK1 and $\mathrm{DCLK}^{+}$tuft cells were decreased in Sox $4 \mathrm{cKO}$ intestines, we sought to examine the regulatory role of Sox4 in tuft cell differentiation more closely. IL-13 is known to induce tuft cell differentiation, and is produced by Group 2 innate lymphoid cells (ILC2) that are recruited to the intestinal epithelium in response to tuft cell secreted IL-25. This mechanism produces an extrinsic feed-forward loop that signals through unknown intrinsic response elements in ISCs and progenitors to drive tuft cell hyperplasia following parasitic helminth infection ${ }^{2,5}$. We treated wild type intestinal organoids with IL-13 to determine its effect on Sox4. As previously reported, IL-13 induced expression of tuft cell-specific Dclk1 (Supplementary Figure 7A). Compellingly, IL-13 also induced a dose-dependent upregulation of Sox4, driving an approximately 3-fold increase at the optimal dose (Supplementary Figure 7A). Levels of Sox4 upregulation correlated closely 
with Dclk1 induction. As expected, goblet cell Muc2 was the only non-tuft lineage specific gene also upregulated in response to IL-13 (Supplementary Figure 7B) ${ }^{2}$. To determine if Sox4 is required for proper Dclk1 upregulation in response to IL-13, we next treated Sox $4 \mathrm{cKO}$ and control organoids with IL-13. While control organoids exhibited robust induction of Dclkl and the tuft cell transcription factor Pou2f3, Sox4cKO organoids failed to significantly upregulate these genes (Figure 4A).

Next, we asked if Sox4-driven tuft cell responses were physiologically necessary for clearance of helminth infection. Sox $4 \mathrm{cKO}$ and control animals were infected with $N$. brasiliensis, a helminth known to induce tuft cell-mediated type II immune reactions ${ }^{2,4}$, 5. Intestines were examined 7 days post-infection (d.p.i.), at the peak of tuft cell hyperplasia, when most worms are typically cleared from wild type mice ${ }^{5}$. We first examined SOX $4^{+}$cells in control mice. Helminth infection drove a 4-fold increase in the number of SOX $4^{+}$cells per crypt (Figure 4B). As expected, DCLK $1^{+}$cell numbers were largely upregulated in control animals (Figure 4C). In contrast, Sox4cKO intestines exhibited an attenuated increase in tuft cell numbers (Figure 4C). To determine if impaired tuft cell hyperplasia in Sox4cKO animals affected worm burden at 7d.p.i., we quantified the number of adult worms present in the intestines of infected animals. In order to match worm clearance data with tuft cell hyperplasia in the same mouse, duodenal and ileal tissues were harvested to establish worm counts and jejunal tissues were used for histological studies, discussed above. Control animals exhibited a neartotal clearance of adult worms, while Sox $4 \mathrm{cKO}$ animals bore a significantly higher worm burden at 7d.p.i. (Figure 4D). These data implicate Sox4 as a regulator of tuft cell differentiation at physiological baseline, with an important role in tuft cell hyperplasia during helminth infection.

\section{Sox4- and Atoh1-expressing cells form overlapping and distinct populations}

Early tuft cell specification remains a paradox in intestinal epithelial differentiation. Atohl is accepted as the master regulator of early secretory fate in the intestine, and is de-repressed following loss of Notch signaling ${ }^{36}$. While tuft cells fail to differentiate properly in the presence of constitutively active Notch, multiple lines of evidence demonstrate that a majority of tuft cells are not derived from Atohl-expressing progenitors ${ }^{3,6}$. These data suggest the existence of Notch-repressed, Atohl-independent tuft cell regulatory pathways that remain uncharacterized. Since our data demonstrate that Sox4 is upregulated following Notch inhibition and is involved in tuft cell differentiation, we hypothesized that it might be expressed in an Atohl-independent manner. We examined SOX4 expression in Atoh $1^{\text {GFP }}$ intestines, and found that $55.3 \pm 6.1 \%$ of SOX $4^{\text {high }}$ cells were Atoh1-negative, with the remainder co-localizing with Atoh1-GFP (Figure 5A).

To examine Sox4 and Atohl populations more thoroughly, we reanalyzed previously published single cell RNA-seq (scRNA-seq) data from primary mouse intestinal epithelial cells ${ }^{37}$. Cells were visualized by tSNE dimensionality reduction and classified into previously characterized phenotypic populations (Figure $5 \mathrm{~B}$ ) ${ }^{37}$. Next, we queried the distribution of cell types within each of three populations of interest: (1) Sox4+:Atoh1- $(n=617$ cells), (2) Sox4+:Atoh1+ $(n=88$ cells $)$, and (3) Sox4-:Atoh1+ $(n$ $=162$ cell). Within the Sox $4+$ population, $12.5 \%$ of cells were also Atoh1+. Next, we determined the cellular composition of each of our populations of interest. The 
Sox4+:Atoh1- population consisted primarily of ISCs and progenitor cells (88\%), but also contained the highest proportion of tuft cells relative to other populations (Figure 5C). The Sox 4+:Atoh1+ population was a mix of ISCs and secretory cell types, and contained the highest proportion of EECs relative to other populations (Figure 5C). As expected, the Sox4-:Atoh1+ population was comprised mostly of secretory cells, with the lowest proportion of ISCs an progenitor cells and the highest proportion of goblet and Paneth cells relative to other populations (Figure 5C). These data demonstrate that Sox4 and Atoh1 form distinct and overlapping populations, and that Sox4 is most strongly correlated with ISCs, progenitors, and tuft cells when expressed exclusively of Atohl and more strongly correlated with EECs when co-expressed with Atoh1.

\section{Sox4 drives tuft cell differentiation independently of Atoh1}

We next sought to determine if Sox 4 is sufficient to drive secretory EEC and tuft cell differentiation. To this end, we generated stable transgenic organoids carrying a cumate-inducible Sox 4 overexpression vector (Sox4OE). Gain of function was validated by immunofluorescent detection of SOX4 in empty vector control and induced Sox4OE organoids (Supplementary Figure 8). To validate organoid models for assessment of Sox4-dependent differentiation phenotypes, we included organoids isolated from Sox $4 \mathrm{cKO}$ mice in our analyses. As expected, Sox4cKO organoids exhibited very low numbers of DCLK1+ tuft cells and CHGA+ EECs (Figure 6A, B). In contrast, overexpression of Sox 4 resulted in a significant increase in numbers of both tuft cells and EECs, relative to empty vector controls (Fig 6A, B).

Since scRNA-seq demonstrated differential distribution of Sox4 and Atoh1 across progenitors and secretory cell types, we hypothesized that Sox4 may function independently of Atohl in secretory differentiation. To test this, we generated Sox4OE organoids derived from Atoh $1^{\mathrm{fl} / \mathrm{fl}}$ : vilCre $^{\mathrm{ER}}$ mice. Atohl recombination was induced by treating organoids with $1 \mathrm{uM} 4-\mathrm{OHT}$ once a day for three days and collecting for analysis $24 \mathrm{hr}$ following final treatment with 4-OHT. Analysis of Atohl expression confirmed the effectiveness of this strategy in generating AtohliKO organoids (Figure 6C). To assess whether residual ATOH1 protein was exerting continued effects in Atoh1iKO organoids, we next analyzed $M u c 2$ expression, which is associated with Atoh1-dependent goblet cell differentiation. We found that $M u c 2$ levels were also significantly downregulated in Atoh1iKO organoids, suggesting no residual ATOH1 function at the time of organoid analysis (Figure 6D).

Next, we examined the effect of Atohl and Sox 4 on tuft and EEC differentiation. Surprisingly, Dclk1 was significantly downregulated following loss of Atohl, in direct conflict with reported in vivo data demonstrating an increase in tuft cells in Atohl-null intestines (Figure 6E) ${ }^{10}$. However, treatment of Atoh1iKO organoid cultures with IL13 restored Dclk1 expression to control levels, in contrast to the loss of IL13 responsiveness in Sox4cKO organoids (Supplementary Figure 9). Overexpression of Sox4 was also sufficient to restore Dclk1 expression levels in the absence of Atohl (Figure 6E). Unexpectedly, Chga expression levels remained unchanged following loss of Atoh1, and were upregulated by Sox 4 in AtohliKO organoids (Figure 6F). Notably, Sox4OE failed to rescue or upregulate $M u c 2$ expression levels, suggesting specificity to tuft and EEC lineages (Figure 6D). To refine these observations, we conducted whole mount staining on AtohliKO and Sox4OE organoids and directly quantified tuft and EEC numbers in 
whole organoids. Quantification by immunofluorescence recapitulated gene expression results, demonstrating upregulation of DCLK1+ tuft cells and CHGA+ EECs in Sox4OE organoids regardless of Atoh1 expression (Figure 6G and H). Together, these data demonstrate that Sox4 is sufficient to drive an increase in tuft and EEC differentiation and can do so independently of Atoh1.

\section{Discussion}

Here we show that genetic ablation of Sox 4 in the intestinal epithelium results in increased numbers of proliferating cells and Paneth cells, as well as a reduction in EEC and tuft cell lineages. Our study supports Sox 4 mediated regulation of stem cells in other organ systems by demonstrating that Sox4 regulates ISC function and differentiation toward EECs and tuft cells in the intestinal epithelium. Additionally, we demonstrate that Sox4 has an essential role in pushing lineage allocation toward tuft cell differentiation in response to helminth infection, implying an important role for Sox 4 in host responses to pathogens.

Our group and others have reported Sox 4 expression in Lgr5+ CBCs, but a role for Sox 4 in ISCs has yet to be established ${ }^{18,22}$. We demonstrate that the numbers of ISCs quantified by OLFM4 immunostaining are unchanged between Sox4cKO and control samples. These data suggest that the associated increase in proliferating cells in Sox $4 \mathrm{cKO}$ crypts is likely due to increased progenitor numbers and not more robust ISC activity. Rather, in vitro assay of ISC function demonstrated impaired organoid-forming efficiency of single ISCs isolated from Sox $4 \mathrm{cKO}$ mice. As this dysfunction is not associated with a change in the number of apoptotic cells between control and Sox $4 \mathrm{cKO}$ mice, it is likely that decreased organoid-forming efficiency is caused by loss of stemness and premature differentiation in Sox 4 deficient ISCs. In this regard, Sox4cKO mice demonstrate reduced $\operatorname{Lgr} 5$ mRNA. Lgr5 is highly expressed in ISCs and serves as a receptor for R-Spondin ligands that potentiate Wnt-signaling, which is in turn required to maintain stemness ${ }^{38}$. These data indicate that Sox4 is not required for maintaining ISC numbers, but suggests that Sox4 plays a role in maintaining stemness by positively regulating Lgr5 levels. Functionally, this role would be consistent with Sox4 knockout phenotypes in other tissues, including the developing heart, skeletal muscle, nervous, renal, and hematopoietic systems, which often exhibit precocious differentiation and reduced progenitor and post-mitotic cell numbers $19,20,27,39-41$.

Sox $4 \mathrm{cKO}$ intestines also demonstrate decreased EEC and tuft cell numbers, implying that Sox4 plays a key role in the specification of these secretory lineages. RNAseq analysis demonstrates that Sox4 regulates the expression of the EEC transcription factors, including Isll, Pax6, and Nkx2-2. Isll deletion in the intestine is known to lead to loss of EECs subtypes expressing GIP, CCK, SST, GCG, PYY, and GLP-2 ${ }^{33}$. We demonstrate that loss of Sox4 results in a marked reduction of Isll expression with a concomitant reduction in the numbers of the same EEC subtypes. Together, these results indicate that Sox4 likely functions upstream of EEC transcription factors Isl1, Pax6, and $N k x 2-2$ to drive EEC specification.

The mechanisms that give rise to the tuft cell lineage are largely understudied due to the relatively new characterization of tuft cells as a distinct lineage. The role of intestinal tuft cells was recently described by a handful of independent studies, all of which demonstrated tuft cell hyperplasia following infection with parasitic helminths. 
Following infection, tuft cells initiate a type II immune response through ILC-2 cells 2,4 , 5 . ILC-2 cells secrete IL-13, which induces tuft cell hyperplasia ${ }^{2,4,5}$. Pou $2 f 3$ is known to be essential for tuft cell differentiation, but is also expressed by mature, villus-based tuft cells ${ }^{2}$. Other transcription factors involved in tuft cell specification and hyperplasia remain unknown, and tuft cell progenitors have no distinct biomarkers ${ }^{2}$.

Though SOX $4^{\text {high }}$ cells are relatively rare in homeostasis, we observed a striking increase in SOX $4^{\text {high }}$ cell numbers coincident with peak tuft cell hyperplasia following parasitic infection, suggesting that $\mathrm{SOX} 4^{\text {high }}$ cells may represent a tuft progenitor population. We show that Sox $4 \mathrm{cKO}$ epithelium demonstrates attenuated tuft cell hyperplasia, both in response to recombinant IL-13 in vitro and helminth infection in vivo, and that Sox $4 \mathrm{cKO}$ mice are functionally impaired at clearing worms by 7 d.p.i. Notably, IL13-mediated activation of Pou $2 f 3$ is also impaired in Sox4cKO organoids, suggesting that Sox 4 acts upstream of this established tuft cell transcription factor. Since there is no reporter mouse model for Sox4, we leveraged existing scRNA-seq data to further interrogate the identity of Sox4+ cells. In agreement with histological data, we find that Sox4 is enriched in ISC, progenitor, and secretory populations. Classifying Sox $4+$ cells by Atoh 1 expression reveals a strong correlation between Sox4+:Atoh 1- cells and tuft cells and between Sox4+:Atoh1+ cells and EECs, supporting dual roles for Sox 4 in tuft and EEC differentiation. Functionally, this role is demonstrated by the ability of ectopic Sox4 expression to induce increased tuft and EEC numbers in organoids.

Lineage specification of tuft cells from the ISC/progenitor pool remains a significant question in the field, as tuft cells are capable of being produced independently of Atoh1, but are inhibited by constitutive Notch signaling ${ }^{3,6}$. Further, it was recently shown that inducible knockout of Atohl in vivo leads to increased tuft cell numbers, implying that Atohl may suppress tuft cell differentiation ${ }^{10}$. Surprisingly, our in vitro analyses of Atoh1iKO organoids demonstrate the opposite effect. That is, loss of Atoh1 in vitro leads to decreased tuft cell numbers. However, in contrast to Sox4cKO organoids, which fail to upregulate Dclk1 in response to exogenous IL13, Atoh1iKO organoids retain IL13-responsiveness in terms of Dclk1 expression. These data suggest that tuft cells may be specified by multiple signaling events and pathways, but that IL13-mediated tuft cell hyperplasia is Sox4-dependent and Atohl-independent. In agreement, our gainof-function studies reveal that, although AtohliKO organoids exhibit reduced tuft cell numbers relative to non-recombined controls, Atoh1 is dispensable for Sox4-driven tuft cell differentiation.

A surprising result of our studies is the observation that Sox 4 overexpression was sufficient to drive increased EEC differentiation in the absence of Atoh1. This effect does not appear to be the result of residual ATOH1 activity or non-specific Sox 4 regulation of all secretory differentiation, as goblet cell-associated $M u c 2$ expression remains severely downregulated in Atoh1iKO organoids at the timepoint examined, regardless of Sox4 expression status. These data raise interesting questions regarding the role of Atohl as a master regulator of secretory differentiation. To our knowledge, no other studies to date have expressed transcriptional regulators of secretory differentiation that are normally expressed upstream of Atoh1 in the absence of Atoh1. Further studies in this area would be necessary to determine if other transcription factors are capable of rescuing secretory differentiation in AtohliKO models.

The existence of Atohl-independent lineage specification of tuft cells and EECs 
challenges the long-standing dogma of Atohl as the master transcriptional regulator of secretory differentiation in the intestine. Our findings demonstrate that Sox4 is upregulated following downregulation of Notch or in response to IL13, and drives tuft or EEC specification in a manner that can proceed independently of Atohl. These findings implicate Sox4 as an important regulator of host responses parasitic infection and shed further light on epithelial components of Type II immune responses.

Acknowledgments: The authors would like to thank Drs. Rashmi Chandra and Roger Liddle (Duke University, Durham, NC) for providing antibodies against CCK and PYY; Dr. Veronique Lefebvre for providing Sox $4^{f l}$ mice; Brian Golitz and Noah Sciaky for assistance with Illumina sequencing and analysis; Dr. Pablo Ariel and the UNC Microscopy Services Laboratory for assistance with confocal microscopy. RNA-seq data associated with this manuscript have been deposited in NCBI's Gene Expression Omnibus and are available under GEO accession number GSE90795. This work was funded by the National Institutes of Health, R01 DK091427 (Magness), the Center for Gastrointestinal Biology and Disease P30 DK034987 (Magness), National Institutes of Health, R01 AI1 19004 (Reinhardt), and National Institutes of Health, R01 CA1428260, R01 DK092306 (Shroyer). A.D.G. was partially supported by the UNC GI Division Basic Science Training Grant, T32 DK007737-20 (Sartor), National Institutes of Health, K01 DK111709 (Gracz), and a Research Scholar Award from the American Gastroenterological Association (Gracz).

\section{References}

1. Gracz AD, Magness ST. Defining hierarchies of stemness in the intestine: evidence from biomarkers and regulatory pathways. Am J Physiol Gastrointest Liver Physiol 2014;307:G260-73.

2. Gerbe F, Sidot E, Smyth DJ, et al. Intestinal epithelial tuft cells initiate type 2 mucosal immunity to helminth parasites. Nature 2016;529:226-30.

3. Gerbe F, van Es JH, Makrini L, et al. Distinct ATOH1 and Neurog3 requirements define tuft cells as a new secretory cell type in the intestinal epithelium. J Cell Biol 2011;192:767-80.

4. Howitt MR, Lavoie S, Michaud M, et al. Tuft cells, taste-chemosensory cells, orchestrate parasite type 2 immunity in the gut. Science 2016;351:1329-33.

5. von Moltke J, Ji M, Liang HE, et al. Tuft-cell-derived IL-25 regulates an intestinal ILC2-epithelial response circuit. Nature 2016;529:221-5.

6. Bjerknes M, Khandanpour C, Moroy T, et al. Origin of the brush cell lineage in the mouse intestinal epithelium. Dev Biol 2012;362:194-218.

7. Noah TK, Shroyer NF. Notch in the intestine: regulation of homeostasis and pathogenesis. Annu Rev Physiol 2013;75:263-88.

8. VanDussen KL, Carulli AJ, Keeley TM, et al. Notch signaling modulates proliferation and differentiation of intestinal crypt base columnar stem cells. Development 2012;139:488-97.

9. Westphalen CB, Asfaha S, Hayakawa Y, et al. Long-lived intestinal tuft cells serve as colon cancer-initiating cells. J Clin Invest 2014;124:1283-95. 
10. Herring CA, Banerjee A, McKinley ET, et al. Unsupervised Trajectory Analysis of Single-Cell RNA-Seq and Imaging Data Reveals Alternative Tuft Cell Origins in the Gut. Cell Syst 2017.

11. Gracz AD, Magness ST. Sry-box (Sox) transcription factors in gastrointestinal physiology and disease. Am J Physiol Gastrointest Liver Physiol 2011;300:G50315.

12. Sarkar A, Hochedlinger K. The sox family of transcription factors: versatile regulators of stem and progenitor cell fate. Cell Stem Cell 2013;12:15-30.

13. Formeister EJ, Sionas AL, Lorance DK, et al. Distinct SOX9 levels differentially mark stem/progenitor populations and enteroendocrine cells of the small intestine epithelium. Am J Physiol Gastrointest Liver Physiol 2009;296:G1108-18.

14. Gracz AD, Ramalingam S, Magness ST. Sox9 expression marks a subset of CD24-expressing small intestine epithelial stem cells that form organoids in vitro. Am J Physiol Gastrointest Liver Physiol 2010;298:G590-600.

15. Bastide P, Darido C, Pannequin J, et al. Sox9 regulates cell proliferation and is required for Paneth cell differentiation in the intestinal epithelium. J Cell Biol 2007; 178:635-48.

16. Mori-Akiyama Y, van den Born M, van Es JH, et al. SOX9 is required for the differentiation of paneth cells in the intestinal epithelium. Gastroenterology 2007;133:539-46.

17. Lin CM, Fang CL, Hseu YC, et al. Clinical and Prognostic Implications of Transcription Factor SOX4 in Patients with Colon Cancer. PLoS One 2013;8:e67128.

18. Munoz J, Stange DE, Schepers AG, et al. The Lgr5 intestinal stem cell signature: robust expression of proposed quiescent ' +4 ' cell markers. EMBO J 2012;31:3079-91.

19. Huang J, Arsenault M, Kann M, et al. The transcription factor Sry-related HMG box-4 (SOX4) is required for normal renal development in vivo. Dev Dyn 2013;242:790-9.

20. Potzner MR, Tsarovina K, Binder E, et al. Sequential requirement of Sox4 and Sox11 during development of the sympathetic nervous system. Development 2010;137:775-84.

21. Wilson ME, Yang KY, Kalousova A, et al. The HMG box transcription factor Sox 4 contributes to the development of the endocrine pancreas. Diabetes 2005;54:3402-9.

22. Gracz AD, Williamson IA, Roche KC, et al. A high-throughput platform for stem cell niche co-cultures and downstream gene expression analysis. Nat Cell Biol 2015;17:340-9.

23. Roche KC, Gracz AD, Liu XF, et al. SOX9 Maintains Reserve Stem Cells and Preserves Radio-resistance in Mouse Small Intestine. Gastroenterology 2015.

24. Van Landeghem L, Santoro MA, Krebs AE, et al. Activation of two distinct Sox9-EGFP-expressing intestinal stem cell populations during crypt regeneration after irradiation. Am J Physiol Gastrointest Liver Physiol 2012;302:G1111-32.

25. Buczacki SJ, Zecchini HI, Nicholson AM, et al. Intestinal label-retaining cells are secretory precursors expressing Lgr5. Nature 2013;495:65-9. 
26. Ramalingam S, Daughtridge GW, Johnston MJ, et al. Distinct levels of Sox9 expression mark colon epithelial stem cells that form colonoids in culture. Am $\mathrm{J}$ Physiol Gastrointest Liver Physiol 2012;302:G10-20.

27. Schilham MW, Oosterwegel MA, Moerer P, et al. Defects in cardiac outflow tract formation and pro-B-lymphocyte expansion in mice lacking Sox-4. Nature 1996;380:711-4.

28. el Marjou F, Janssen KP, Chang BH, et al. Tissue-specific and inducible Cremediated recombination in the gut epithelium. Genesis 2004;39:186-93.

29. Penzo-Mendez A, Dy P, Pallavi B, et al. Generation of mice harboring a Sox4 conditional null allele. Genesis 2007;45:776-80.

30. van der Flier LG, van Gijn ME, Hatzis P, et al. Transcription factor achaete scutelike 2 controls intestinal stem cell fate. Cell 2009;136:903-12.

31. Yin X, Farin HF, van Es JH, et al. Niche-independent high-purity cultures of Lgr5+ intestinal stem cells and their progeny. Nat Methods 2014;11:106-12.

32. Desai S, Loomis Z, Pugh-Bernard A, et al. Nkx2.2 regulates cell fate choice in the enteroendocrine cell lineages of the intestine. Dev Biol 2008;313:58-66.

33. Terry NA, Walp ER, Lee RA, et al. Impaired enteroendocrine development in intestinal-specific Islet1 mouse mutants causes impaired glucose homeostasis. Am J Physiol Gastrointest Liver Physiol 2014;307:G979-91.

34. Kobielak K, Stokes N, de la Cruz J, et al. Loss of a quiescent niche but not follicle stem cells in the absence of bone morphogenetic protein signaling. Proc Natl Acad Sci U S A 2007; 104:10063-8.

35. van de Wetering M, Sancho E, Verweij C, et al. The beta-catenin/TCF-4 complex imposes a crypt progenitor phenotype on colorectal cancer cells. Cell 2002;111:241-50.

36. Yang Q, Bermingham NA, Finegold MJ, et al. Requirement of Math1 for secretory cell lineage commitment in the mouse intestine. Science 2001;294:2155-8.

37. Haber AL, Biton M, Rogel N, et al. A single-cell survey of the small intestinal epithelium. Nature 2017;551:333-339.

38. Carmon KS, Gong X, Lin Q, et al. R-spondins function as ligands of the orphan receptors LGR4 and LGR5 to regulate Wnt/beta-catenin signaling. Proc Natl Acad Sci U S A 2011;108:11452-7.

39. Bergsland M, Werme M, Malewicz M, et al. The establishment of neuronal properties is controlled by Sox4 and Sox11. Genes Dev 2006;20:3475-86.

40. Jang SM, Kim JW, Kim D, et al. Sox4-mediated caldesmon expression facilitates differentiation of skeletal myoblasts. J Cell Sci 2013;126:5178-88.

41. Sun B, Mallampati S, Gong Y, et al. Sox4 is required for the survival of pro-B cells. J Immunol 2013;190:2080-9. 


\section{Figures}
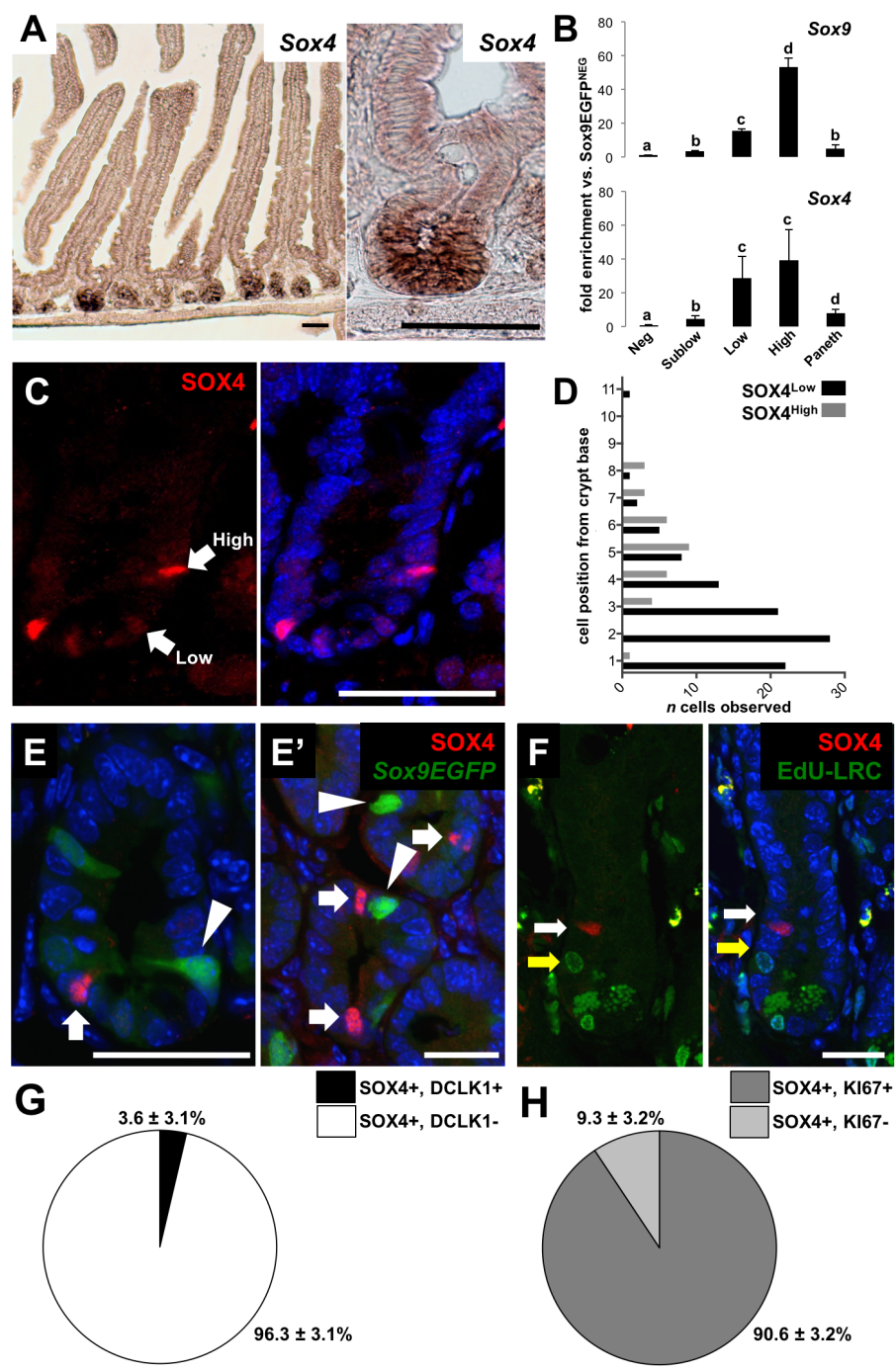

Figure 1. Sox4 is expressed in the ISC and early progenitor zone. (A) In situ hybridization localizes Sox 4 mRNA to the base of small intestinal crypts. (B) FACS isolation of $\operatorname{Sox} 9^{E G F P}$ populations demonstrates enrichment of Sox4 in active ISCs $\left(\operatorname{Sox} 9^{\text {low }}\right)$ and secretory progenitors/reserve ISCs $\left(\operatorname{Sox} 9^{\text {high }}\right)$ (different letters indicate statistically significant differences, $\mathrm{p}<0.05$ ). (C) SOX4 protein is expressed in the ISC and supra-Paneth cell zones at variable levels, with (D) SOX $4^{\text {low }}$ cells primarily localized to the CBC position (1-3) and SOX $4^{\text {high }}$ cells occurring mainly in the supra-Paneth cell zone (4+). (E/E') SOX $4^{\text {high }}$ and Sox $9^{\text {high }}$ cells do not co-localize; arrows indicate SOX4hi cells and arrowheads indicate Sox $9^{\text {high }}$ cells $\left(\mathrm{n}=3\right.$ mice, $100 \mathrm{SOX} 4^{\text {high }}$ cells per mouse, scale bar represents $20 \mathrm{um}$ ). (F) SOX $4{ }^{\text {high }}$ cells are not LRCs by $28 \mathrm{~d}$ continuous EdU labeling followed by $10 \mathrm{~d}$ washout; white arrow indicates SOX $4{ }^{\text {high }}$ cell and yellow arrow indicates LRC (scale bar represents 20um; note: EdU detection kit reacts with Paneth cell granules). (G) A small portion of SOX $4^{\text {high }}$ cells co-localize with the tuft cell marker DCLK1 and $(\mathrm{H})$ a majority of SOX $4^{\text {high }}$ cells are positive for proliferative marker KI-67. 


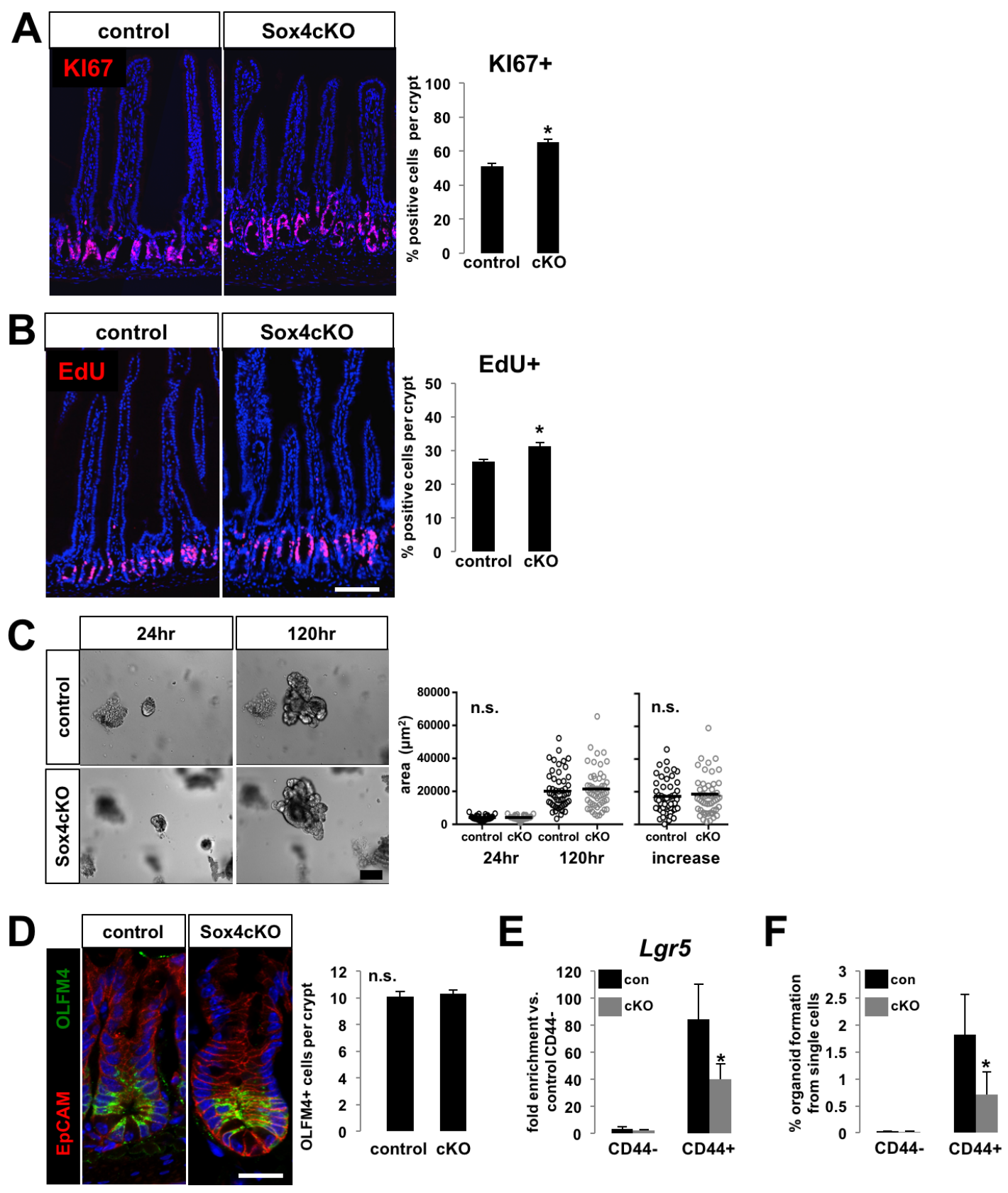

Figure 2. Loss of Sox4 results in increased proliferation and decrease in functional stemness, with no change in ISC numbers. (A) Sox 4cKO intestines exhibit increased total proliferation by KI67 staining, as well as (B) increased numbers of cells in S-phase, as indicated by EdU (scale bar represents 100um). (C) Whole crypts isolated from Sox 4cKO intestines form organoids that undergo similar growth rates to control crypts (scale bar represents 100um). (D) Numbers of OLFM4+ ISCs remain unchanged in Sox4cKO crypts, while (E) Lgr5 is downregulated in CD44+ populations from Sox4cKO intestines. Functional stemness, as assayed by organoid forming ability, is significantly decreased in CD44+ populations isolated from Sox $4 \mathrm{cKO}$ intestines (scale bar represents 25um; asterisks indicate significance; $p<0.05$ ). 

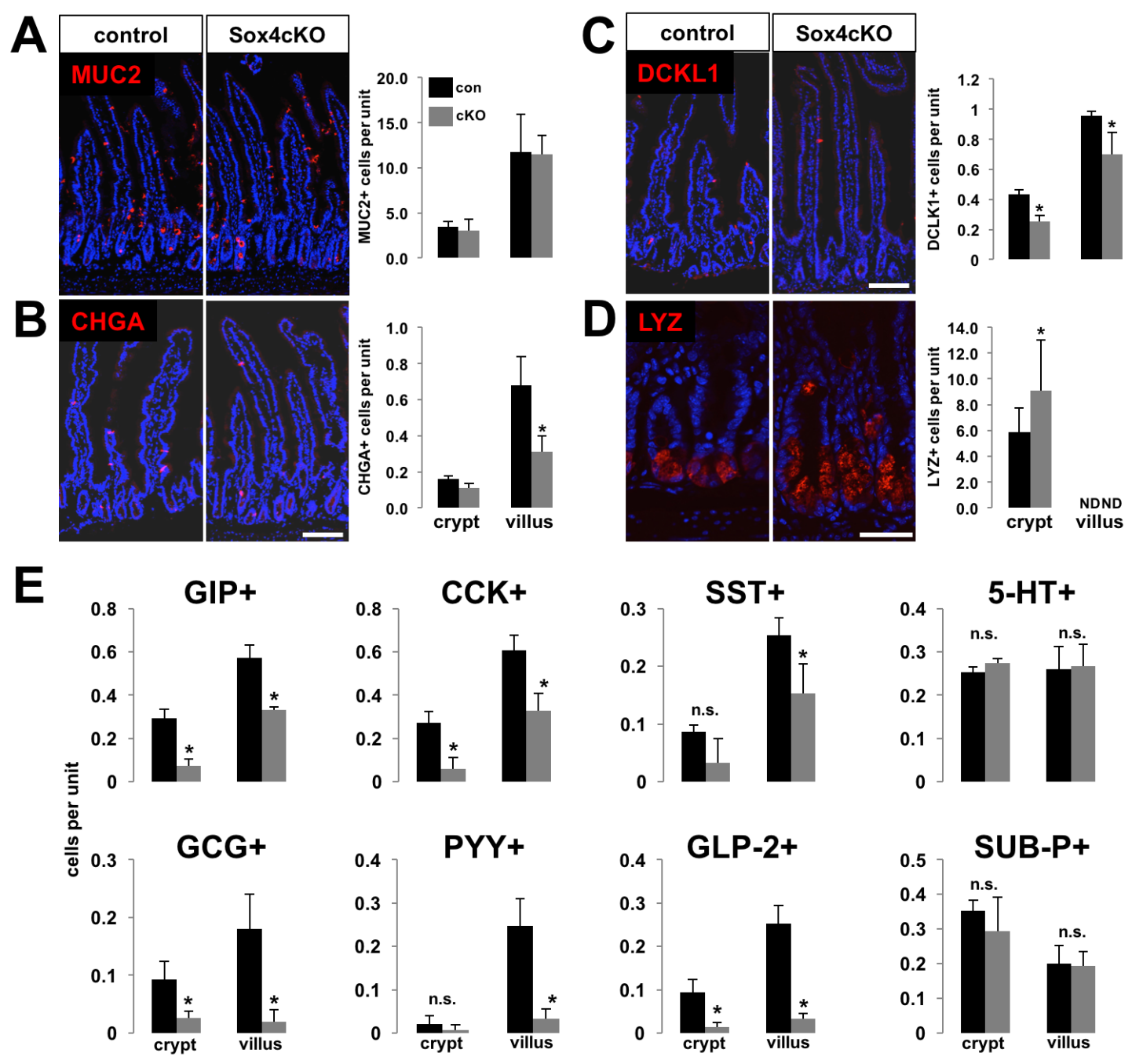

Figure 3. Sox4 is required for proper secretory lineage allocation. (A) MUC2+ goblet cells remain unchanged in Sox4cKO intestines, while (B) CHGA + EECs are found at decreased numbers in Sox4cKO villi, and (C) DCLK1+ tuft cells are decreased in both crypt and villus compartments of Sox $4 \mathrm{cKO}$ intestines (scale bar represents 100um). (D) Paneth cell numbers are increased in Sox4cKO crypts (scale bar represents 50um). (E) Assessment of EEC differentiation by immunofluorescence against markers associated with specific EEC subtypes reveals that Sox4 is required for proper specification of a subset of EEC lineages. Conversely, numbers of cells positive for serotonin (5-HT) and Substance $\mathrm{P}$ (SUB-P) are unaffected in Sox4cKO intestines. (asterisks indicate significance; $\mathrm{p}<0.05)$. 


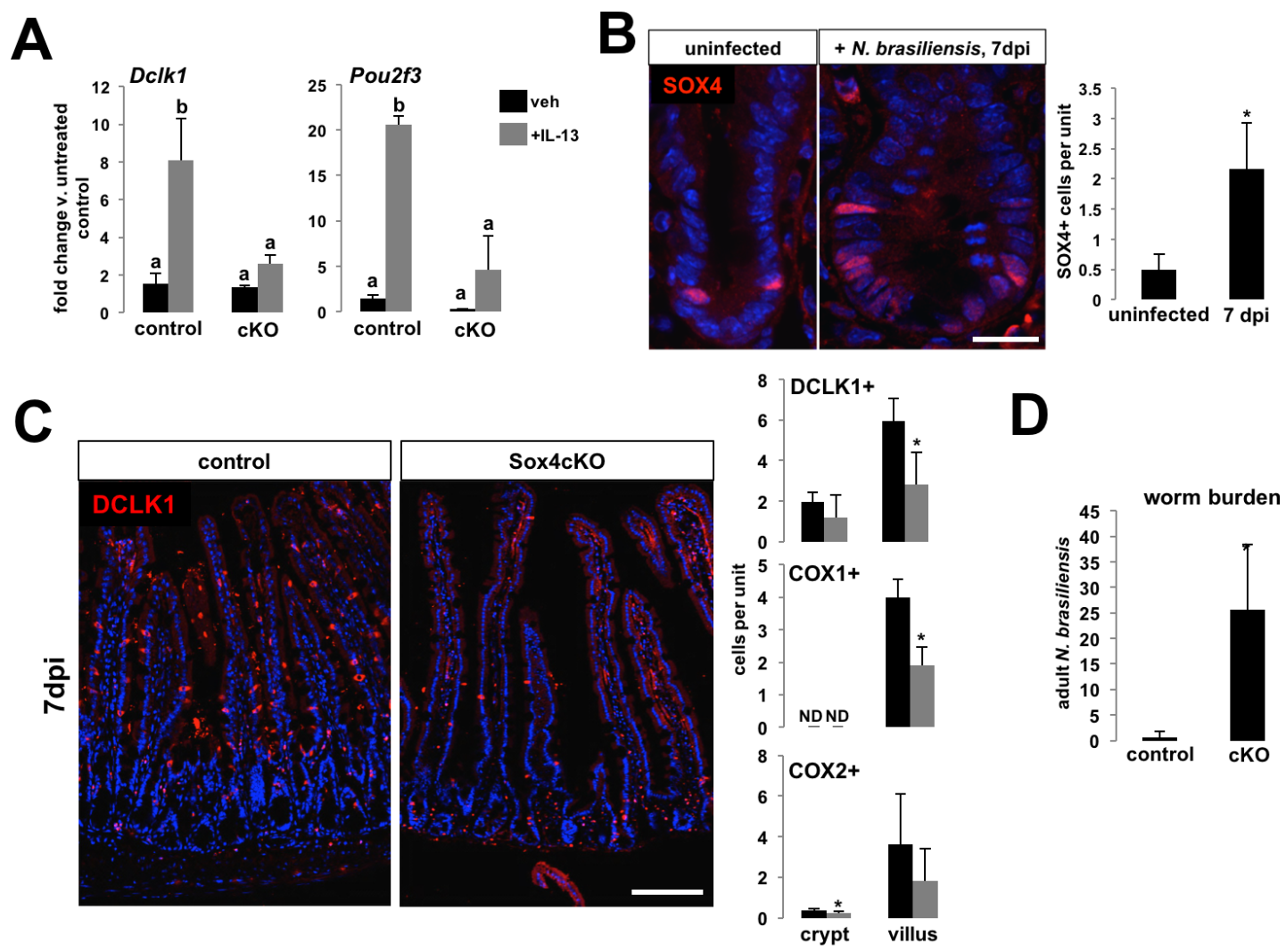

Figure 4. Sox4 regulates tuft cell differentiation and is required for tuft cell hyperplasia following helminth infection. (A) Tuft cell markers Dclk1 and Pou $2 f 3$ are upregulated in control, but not Sox4cKO organoids, in response to treatment with recombinant IL-13 (different letters indicate statistically significant differences, $\mathrm{p}<0.05$ ). (B) 7 days postinfection with $N$. brasiliensis, control intestinal crypts exhibit a significant expansion of SOX $4^{\text {high }}$ cells (scale bar represents $20 \mathrm{um}$ ). (C) Control mice demonstrate expected tuft cell hyperplasia at 7dpi, where Sox4cKO intestines have significantly fewer tuft cells as quantified by DCLK1, COX1, and COX2 (ND = no data; COX1+ cells are not found in intestinal crypts. Scale bar represents $100 \mathrm{um}$ ). (D) Sox $4 \mathrm{cKO}$ mice also fail to fully clear adult worms by $7 \mathrm{dpi}$ (asterisks indicate significance, $\mathrm{p}<0.05, \mathrm{n}=3$ mice per group). 

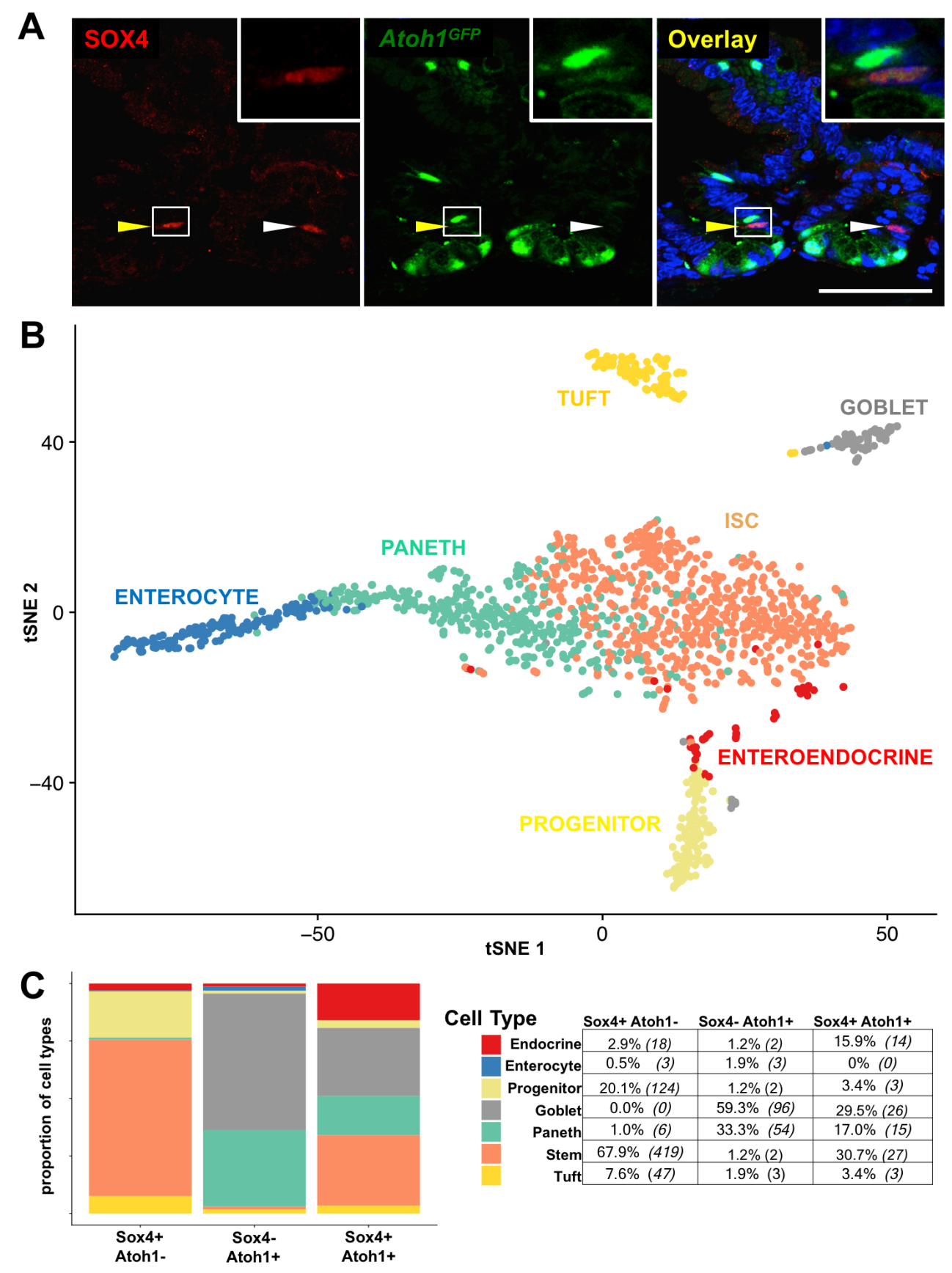

\begin{tabular}{|c|c|c|c|}
\hline ype & Sox4+ Atoh1- & Sox4-Atoh1+ & Sox4+ Atoh1+ \\
\hline Endocrine & $2.9 \%(18)$ & $1.2 \%(2)$ & $15.9 \%(14)$ \\
\hline Enterocyte & $0.5 \% \quad$ (3) & $1.9 \%(3)$ & $0 \%(0)$ \\
\hline Progenitor & r $20.1 \%(124)$ & $1.2 \%(2)$ & $3.4 \%(3)$ \\
\hline Goblet & $0.0 \%(0)$ & $59.3 \%(96)$ & $29.5 \%(26)$ \\
\hline Paneth & $1.0 \%(6)$ & $33.3 \%(54)$ & $17.0 \%(15)$ \\
\hline Stem & $67.9 \%(419)$ & $1.2 \%(2)$ & $30.7 \%(27)$ \\
\hline Tuft & $7.6 \%(47)$ & $1.9 \%(3)$ & $3.4 \%$ (3) \\
\hline
\end{tabular}

Figure 5. Atoh1+ and Sox4+ exhibit overlapping and exclusive expression in secretory cells and ISCs. (A) Co-detection of SOX4 and Atoh1, using a transgenic Atoh1 ${ }^{\text {GFP }}$ allele, reveals that $55.3 \pm 6.1 \%$ of SOX4+ cells co-express Atohl (yellow arrowhead indicates SOX4+/Atoh1+ cell; white arrowhead indicates SOX4+/Atoh1- cell. Scale bar represents 25um). (B) scRNA-seq ${ }^{37}$ identifies unique cell populations in the intestinal epithelium (C) Classifying cells based on Sox4 and Atoh1 expression status (Sox4+:Atoh1-, Sox4:Atoh1+, and Sox4+:Atoh1+) reveals that Sox4+ populations contain the highest proportion of tuft cells, EECs, and ISCs, consistent with observed phenotypes in vivo and in vitro. 


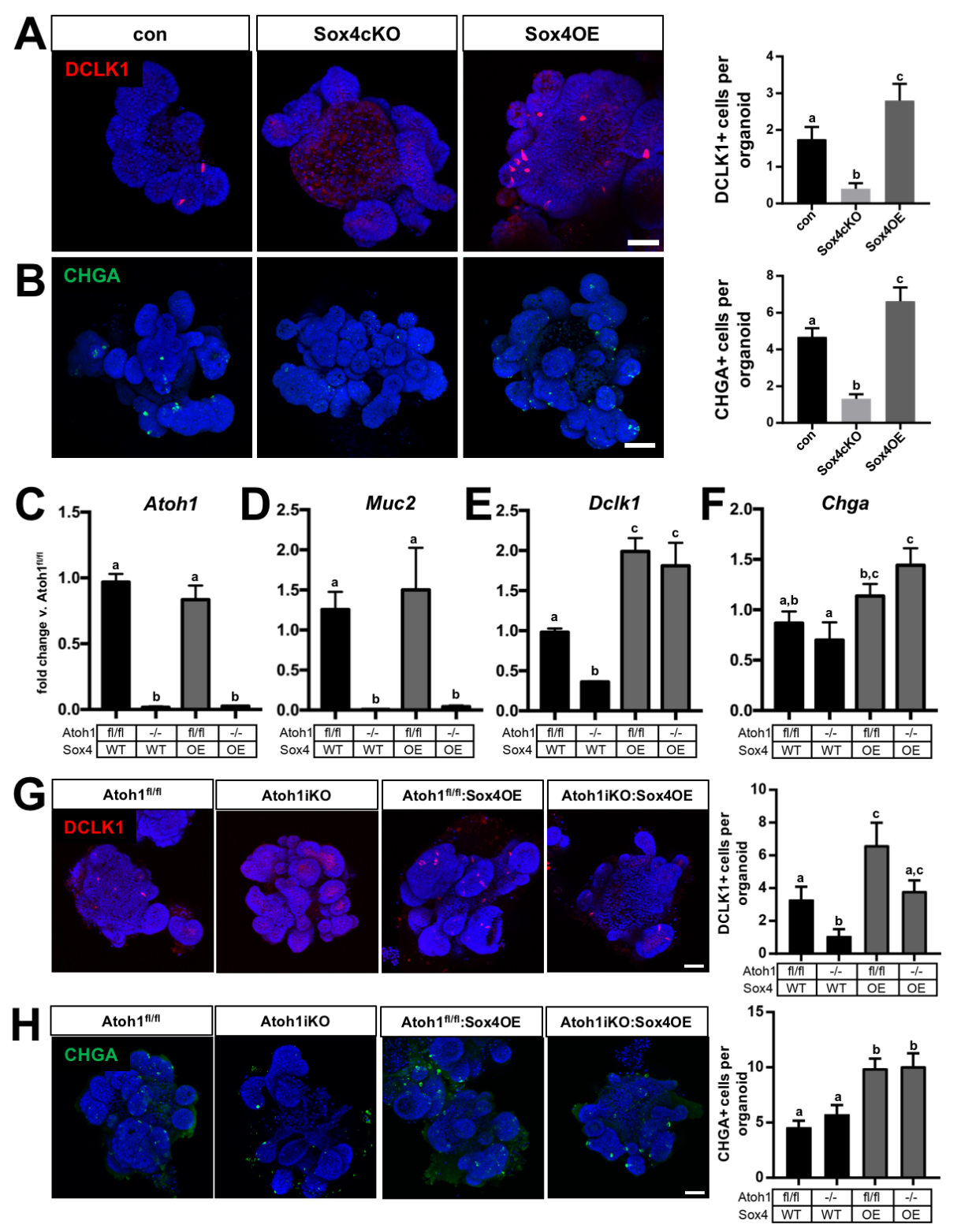

Figure 6. Sox4 drives tuft cell and EEC differentiation independently of Atoh1. (A) Tuft cells, as identified by DCLK1, are rare in control organoids, absent in Sox4cKO organoids, and upregulated in Sox4OE organoids. (B) Similar results are observed for CHGA+ EECs, indicating that Sox4 is sufficient to increase tuft and EEC differentiation. (C) 4-OHT treatment downregulates Atohl in control and Sox4OE organoids, resulting in (D) loss of Muc2 expression, which demonstrates loss of Atohl-driven transcription. (E) Loss of Atohl in organoids also results in downregulation of Dclk1, which is rescued to normal levels in Sox4OE organoids, regardless of Atohl expression. (F) Chga is not affected by Atoh1 deletion in organoids, but is upregulated by Sox4OE in the absence of Atoh1. Cell counts of (G) DCLK1+ tuft cells and (H) CHGA+ EECs demonstrate correlation between changes in gene expression and lineage specification in AtohliKO and Sox4OE organoids. Notably, CHGA+ cells numbers are increased in Sox4OE organoids both with and without Atohl expression. 